\title{
METHANOL TOLERANT OXYGEN REDUCTION ACTIVITY OF NITROGEN- IRON DOPED CARBON IN A HOLLOW CORE MESOPOROUS SHELL STRUCTURE
}

K. Y. Chan and M. Zhou (The University of Hong Kong)

A well-defined porous carbon structure with tunable parameters in different length scales can provide a platform for systematic study of transport and confinement effects on electrochemical conversion. For example, the carbon structure with tunable hierarchical porosity composed of a hollow core within a mesoporous shell (HCMS) is used to investigate transport limitations in electrochemical capacitance [1, 2]. Recently, we synthesized the same HCMS carbon structure with doping of nitrogen and iron and investigated the electrocatalytic activity for oxygen reduction in acid medium [3]. As shown in Fig. 1, the electrocatalyst has a mesoporous shell of $50 \mathrm{~nm}$ thick around a hollow core of $350 \mathrm{~nm}$. The pores have uniform diameter of $8 \mathrm{~nm}$. The synthesis follows the procedure described in [1] and the introduction of iron nitrate and ethylene diamine as nitrogen and iron precursors [3, 4].

Despite having no precious metals, the Fe-N-HCMS electrocatalyst displays excellent performance for oxygen reduction reaction. The better mass transport of the HCMS structure leads to higher diffusion limitation currents compared to other carbon structures. The Fe-N doped HCMS catalyst also performs better at high current than a similar HCMS carbon with Pt nanoparticles but no Fe-N doping. This is attributed to uniformly distributed activity of Fe-N doped carbon as opposed to localized activity $\mathrm{Pt}$ nanoparticles which is vulnerable to diffusion limitation. Furthermore, Fe-N/HCMS carbon electrocatalyst is inactive towards methanol oxidation, thus rendering it ideal for methanol tolerant oxygen reduction. As shown in Fig. 2, platinum containing electrocatalysts has an overall anodic current between above $0.6 \mathrm{~V} / \mathrm{RHE}$ due to methanol oxidation, whereas Fe-N-HCMS carbon is insensitive to presence of methanol. The Fe-NHCMS electrocatalyst also display good durability in accelerated degradation tests (ADTs) of over 1000 cycles of voltage scan between 0.6 and 1.0 V/RHE. Under the same ADTs, Pt containing electrocatalysts all show large increase in overpotential at the end of 1000 cycles.

\section{Acknowledgement}

The work was supported by a grant from Hong Kong Research Grants Council GRF HKU 700209P) and funding from ICEE via SRT Clean energy and UDF of University of Hong Kong.

References:

[1]. F. Li, M. Morris and K.Y. Chan*, J. Mater. Chem.21 (2011) 8880-8886.

[2] C. Yang, C.-Y. V. Li, F. Li, K.-Y. Chan*, .J. Electrochem. Soc. 160(4) (2013) H271$\mathrm{H} 278$.

[3] M. Zhou, C. Yang, and K.Y. Chan, Adv. Energy Mater. DOI:

10.1002/aenm.201400840, Aug 2014.

[3] H. W. Liang, W. Wei, Z. S. Wu, X. Feng, K. Müllen, J. Am. Chem. Soc. 2013, 135, 16002. 
[4] T. Palaniselvam, B. P. Biswal, R. Banerjee, S. Kurungot, Chem. Eur. J. 2013, 19, 9335.

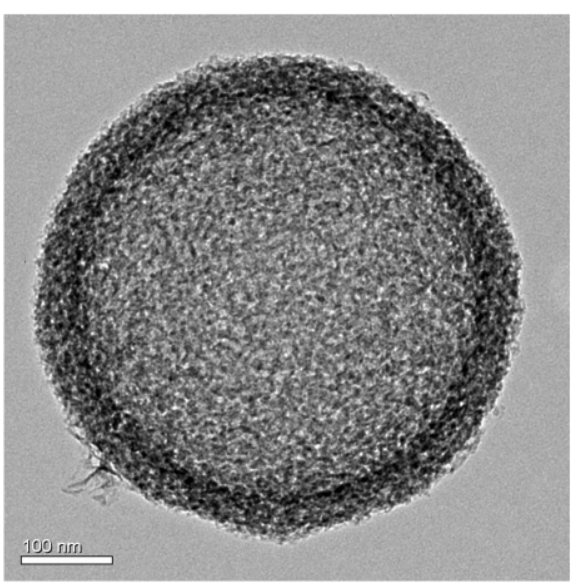

Fig. 1. Transmission electron micrograph of the Fe-N doped carbon with a $350 \mathrm{~nm}$ hollow core and $50 \mathrm{~nm}$ shell region with uniform mesopores of $8 \mathrm{~nm}$, possessing $1199 \mathrm{~m}^{2} / \mathrm{g}$ and $1.77 \mathrm{~cm}^{3} / \mathrm{g}$.

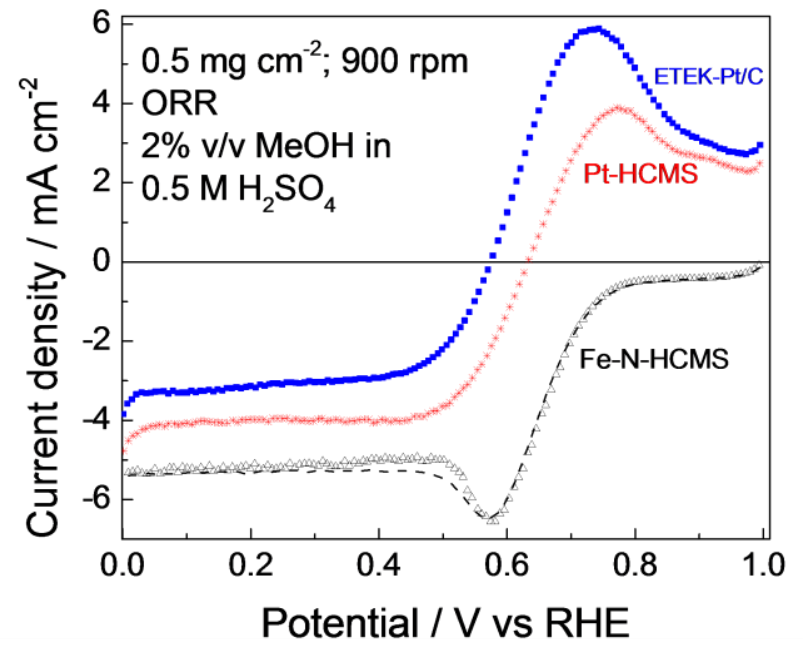

Fig. 2 Polarization of oxygen reduction in presence of $1 \mathrm{~mL}$ methanol per $50 \mathrm{~mL} 0.5 \mathrm{M} \mathrm{H}_{2} \mathrm{SO}_{4}$ at rotation speed of 900 $\mathrm{rpm}$. The solid squares, asterisks, and open triangles are ETEK, Pt-HCMS, and Fe-N-HCMS, at mass loading of 0.5 $\mathrm{mg} / \mathrm{cm}^{2}$, respectively. The dashed line is corresponding ORR curve of Fe-N-HCMS in absence of methanol. 